\title{
Vorwort zur neunten Auflage
}

Nach reichlich fünfzehn Jahren liegt die neunte Auflage des Dornseiff vor Ihnen. In der neuen Auflage sind rund 25\% mehr Wörter verzeichnet. Zusätzlich wurde das Layout überarbeitet, so dass sie einzelnen Sachgruppen durch Hervorhebung wichtiger Wörter übersichtlicher geworden sind. An der logischen Gesamtstrukturstruktur hingegen wurden praktisch keine Veränderungen vorgenommen.

Um das Volumen des Dornseiff nicht weiter zu vergrößern, wurden die bisher enthaltenen Fachaufsätze ausgelagert. Dies betrifft

- die Lexikographisch-Historische Einführung von Herbert Ernst Wiegand,

- die Ausgewählte Bibliographie zur Lexikographie und Onomasiologie von Herbert Ernst Wiegand sowie

- die Wortschatzdarstellung und Bezeichnungslehre von Franz Dornseiff.

Diese Texte sind weiterhin auf der Website des Verlags elektronisch verfügbar unter https://www.degruyter.com/view/title/517003.

Auch bei der neunten Auflage unterstützten Mitarbeite der Abteilung Automatische Sprachverarbeitung sowie Studierende das Projekt. Mein spezieller Dank gilt Lena Schiffer und Gabriel Huppenbauer für die Hilfe bei der automatischen Einordnung der neu aufzunehmenden Wörter in das Sachgruppensystem und bei der manuellen Überprüfung dieser Einordnung. Christopher Schröder besorgte den XML-Export des Sachgruppenteils sowie des alphabetischen Zugriffsregisters.

Uwe Quasthoff

Leipzig, im November 2019

\section{Vorwort zur achten Auflage}

Mit der vorliegenden achten Auflage wird die erste inhaltliche Änderung seit der fünften Auflage 1959 präsentiert. Die mutige Entscheidung des Verlages, die Neubearbeitung einem Informatiker mit Schwerpunkt Automatische Sprachverarbeitung anzuvertrauen, hat nach vierjähriger Arbeit zu einer Neuauflage mit vorsichtig modernisierter Struktur, völlig überarbeitetem Wortschatz und verbesserten Orientierungsmöglichkeiten geführt. 
Die Auswahl der aufgenommenen Wörter und Wortgruppen erfolgte erstmals korpusbasiert: Nicht das Sprachgefühl des Bearbeiters, sondern die Häufigkeit in einer sehr großen Textmenge wurde als Entscheidungskriterium genutzt, ob ein Wort oder eine Wortgruppe aufgenommen wird. Als Ergebnis ist der neue Dornseiff aktuell. Gegenüber der siebten Auflage wurden mehr als 30\% neue Wörter und Wortgruppen aufgenommen. Das Sachgruppensystem wurde um neue Themenbereiche erweitert beispielsweise aus den Bereichen Börse, Computer, Medien, Medizin, Naturwissenschaften, Sport und Verkehr. Bei nahezu gleichem Umfang wie die siebte Auflage enthalten die jetzt 970 Sachgruppen wieder rund 90000 Wörter und Wortgruppen.

Erstmals verfügt der Deutsche Wortschatz nach Sachgruppen über ein vollständiges alphabetisches Zugriffsregister.

Neu sind die Lexikographisch-historische Einführung sowie die Ausgewählte Bibliographie zum Thema „Lexikographie und Onomasiologie“, beides von Herbert Ernst Wiegand. Übernommen aus der siebten Auflage wurde der Artikel Wortschatzdarstellung und Bezeichnungslehre von Franz Dornseiff.

An dieser Stelle möchte ich all denen danken, die diese Neubearbeitung hilfreich und kritisch begleitet haben. Zunächst gilt mein Dank meinen Kollegen von der Abteilung Automatische Sprachverarbeitung am Institut für Informatik der Universität Leipzig, speziell dem Leiter Gerhard Heyer. Hier erhielt ich die nötigen Freiräume sowie wohlwollende Unterstützung in jeder Hinsicht. Für hilfreiche und äußerst anregende Diskussionen zu lexikographischen und metalexikographischen Themen danke ich Herbert Ernst Wiegand (Heidelberg). Dem Verlag, insbesondere seinem Cheflektor Sprachund Literaturwissenschaft, Heiko Hartmann, möchte ich für die konstruktive Zusammenarbeit und die Bereitschaft zur Umsetzung der verschiedenen Gestaltungsvorschläge danken. Weitere Unterstützung erfuhr ich durch Markus Bandur (Freiburg), Ulrike Haß-Zumkehr (Mannheim), Karlheinz Jakob (Dresden), Stefan Schierholz (Göttingen), Ingrid Wiese (Leipzig) und Rüdiger Zymner (Wuppertal) welche die Wörterbuchartikel aus den Bereichen Musik, Politik, Technik, Sport, Medizin und Literatur als fachliche Gutachter kritisch durchsahen.

Technische Unterstützung kam von zwei ehemaligen Studenten. Sandra Liebold begleitete diese Neubearbeitung über alle vier Jahre vom Einscannen der alten Filme bis zu den letzten Korrekturen. Christian Biemann erledigte die Alphabetisierung, das Erstellen des vollständigen alphabetischen Zugriffsregisters und den XML-Export der Daten.

Herzlichster Dank gebührt schließlich meiner Frau Karin und meinem Sohn Matthias, deren Unterstützung ich mir immer sicher sein konnte und die während der „heißen Phase“ der Arbeit alles taten, um mir das Vorankommen zu erleichtern. Ihnen ist dieses Wörterbuch auch gewidmet. 